\title{
Dental Anomalies in Down Syndrome Individuals: A Review
}

\author{
Vanathy Desingu ${ }^{1}$, Amrutha Adapa ${ }^{2}$, Senthil Kumar ${ }^{3}$, Shantha Devi ${ }^{4}$
}

\begin{abstract}
Background and aim: Down syndrome, also denominated as trisomy 21 , is a genetic alteration in which the affected individual carries an extra chromosome 21. Down syndrome patients have specific orofacial features such as mouth breathing, open bite, macroglossia, hypodontia, microdontia, anodontia, fissured tongue, malocclusion, low level of dental caries, poor oral hygiene, delayed eruption, small maxilla, retained deciduous teeth. The present review is aimed to evaluate the prevalence of dental anomalies in Down syndrome individuals.

Materials and methods: The primary focus of the search was review articles, case report and observational studies explaining the common dental anomalies affecting Down syndrome individuals.

Results: One review article, two case reports and twelve observational studies were included to this review. Prevalence of overall dental anomalies in Down syndrome individuals is about 50.47-95.52\% and individual percentage of dental anomalies, taurodontism 9.52-85.71\%; anodontia $34.69 \%$; delayed eruption 2.04\%; conic teeth $14.28 \%$; microdontia $2.04-16.19 \%$; fusion $2.04 \%$; hypodontia $16.19-62 \%$; fissured tongue $78 \%$; retained teeth $10.17 \%$; and teeth agenesis $30-81 \%$ were noticed in Down syndrome individuals.

Conclusion: Individuals with Down syndrome exhibit high prevalence of dental anomalies compared to normal individuals. The most prevalent dental anomalies among these individuals were taurodontism, anodontia, hypodontia, fissured tongue and teeth agenesis. These outcomes reinforce that dental care should be directed to prevent and/or control problems and to improve oral hygiene status of Down syndrome individuals. Keywords: Children, Dental anomalies, Trisomy 21.

Journal of Scientific Dentistry (2019): 10.5005/jp-journals-10083-0902
\end{abstract}

\section{INTRODUCTION}

Down syndrome is the most common chromosomal disorder in newborns and causes delay in physical and intellectual development, ${ }^{1}$ due to the presence of one extra copy of chromosome 21 in their cells. ${ }^{2}$ It is the most common genetic birth defect affecting approximately one in 700 births. Children with Down syndrome are at an increased risk for health complications beyond usual childhood illness and many disorders known to interfere with growth, such as coeliac disease and hypothyroidism. ${ }^{1}$ Clinically, it is characterized by neurological changes, structural cardiopathy, respiratory problems, increased risk of infection, increased risk of leukemia, dental anomalies, orofacial dysmorphology, ${ }^{3}$ bone growth disorders and generalized muscle hypotonia. ${ }^{4}$ The craniofacial and oral features involved in Down syndrome include brachycephaly, usually small nose associated with low nasal bridge, small maxilla, tongue with fissures, papillary hypertrophy ${ }^{5}$ prominent epicanthic folds, hypoplastic cranial base, ${ }^{6,7}$ more hypoplastic facial middle third and smaller lower facial third than non-syndromic patient. ${ }^{5}$

Down syndrome patients have specific orofacial features such as taurodontism, open bite, macroglossia, hypodontia, microdontia, anodontia, drooling, fissured tongue, malocclusion, low level of dental caries, poor oral hygiene, delayed eruption, small maxilla, retained deciduous teeth. ${ }^{3}$

The present review is aimed to evaluate the prevalence of dental anomalies in Down syndrome individuals.

\section{Materials and Methods}

The sources were searched from a database of PubMed from 2007 to 2019 (last 13 years). The inclusion criteria included key words of microdontia, delayed eruption, dental anomalies, taurodontism, retained teeth, anodontia, fissured lip and tongue, macroglossia,
${ }^{1-4}$ Department of Oral Pathology and Microbiology, Sri Balaji Vidyapeeth University (a Deemed University), Pillayarkuppam, Puducherry, India

Corresponding Author: Vanathy Desingu, Department of Oral Pathology and Microbiology, Sri Balaji Vidyapeeth University (a Deemed University), Pillayarkuppam, Puducherry, India, Phone: +91 8940192155, e-mail: amrutha.varshiadapa@gmail.com

How to cite this article: Desingu V, Adapa A, Kumar S, Devi S. Dental Anomalies in Down Syndrome Individuals: A Review. J Sci Dent 2019;9(1):6-8.

Source of support: Nil

Conflict of interest: None

hypodontia in Down syndrome. The primary focus of the search was review articles, case report and observational studies explaining the common dental anomalies affecting Down syndrome individuals.

\section{Result}

The 14 articles that met the selection criteria (1 review article, 2 case reports and 12 observational studies) were included to this review, of which some of the studies revealed that the overall prevalence of dental anomalies in Down syndrome individuals is about $50.47-95.52 \%,{ }^{2,5}$ while the other studies concluded that the prevalence of dental anomalies such as taurodontism $85.71 \%,{ }^{5}$ 9.52\%; ${ }^{2}$ anodontia $34.69 \%{ }^{5}$ delayed eruption $2.04 \% ;{ }^{5}$ conic teeth $14.28 \%{ }^{5}$ microdontia $2.04 \%,{ }^{5} 16.19 \%$; fusion $2.04 \%{ }^{5}$ hypodontia $62 \%,{ }^{1} 16.19 \%{ }^{2}$ fissured tongue $78 \%{ }^{3}$ retained teeth $10.17 \%{ }^{2}$ and teeth agenesis $65 \% ;{ }^{8} 30-81 \%{ }^{9}$ in Down syndrome individuals.

\section{Discussion}

Down syndrome, also denominated as trisomy 21, is a genetic alteration in which the affected individuals carry an extra 
chromosome $21 .{ }^{5}$ It is the most common genetic birth defect. The oral manifestations of Down syndrome include, hypoplasia of the maxilla resulting in microstomia (small mouth), macroglossia, fissured tongue, taurodontism, anodontia, delayed eruption, microdontia, missing/malformed teeth, retained deciduous teeth and bruxism. Maternal age is a significant factor of incidence; the disease occurs in 1 in 1,550 live births in women under 20 years of age, in contrast to 1 in 25 live births in mothers over 45 years. ${ }^{10}$

Prenatal testing is the best strategy for reducing the burden of genetic disorders and congenital disabilities that cause significant postnatal functional impairment. ${ }^{7}$ Prenatal screening for Down syndrome is done in the first trimester between 11 weeks and 14 weeks by ultrasound measurement of nuchal translucency (NT) and the pregnancy associated plasma protein-A (PAPP-A) and free human chorionic gonadotropin (free-beta HCG) biomarkers in maternal serum. ${ }^{11}$ The families at risk are mostly identified after birth of affected child, but many genetic disorders occur in families, even without any previous history of affected child.

With advanced medical science, screening test for genetic disorder are available for all pregnant women in both private and public sectors and awareness amongst obstetricians and primary care physicians is increasing. ${ }^{7}$ However, proper counseling about the test is not popular among the general population, which leads to the birth of children with Down syndrome. ${ }^{7}$ At present, there is no nation-wide consensus regarding the nature and timing of these prenatal screening protocol. Due to the absence of any definite guidelines and the additional lacunae in the awareness regarding the appropriate prenatal screening in the country, the optimum benefits of these screening protocols are not reaching the population. ${ }^{7}$

\section{Dental Anomalies Related to Down Syndrome}

Down syndrome patients have specific orofacial features such as mouth breathing, open bite, macroglossia, fissured lips and tongue, delayed teeth eruption, missing and malformed teeth, microdontia, crowding, over-retained deciduous teeth, bruxism and hypodontia. ${ }^{5}$ The prevalence of dental anomalies in patients with Down syndrome patients is about $50.47-95.5 \%$. $^{2,5}$

The high prevalence of dental anomalies in individuals with Down syndrome is associated with slow cellular growth rhythm and reduction in number of cells that shows strong influence on the teeth development, associated with hypodontia, microdontia, taurodontism. ${ }^{2}$

Taurodontism was the most common dental anomaly seen in Down syndrome patients. Moraes et al. investigated the incidence of taurodontism in 49 subjects with Down syndrome and it was found in 42 individuals with 238 involved teeth (85.71\%). ${ }^{5}$ Cuoghi et al. evaluated 105 patients with Down syndrome and $9.52 \%$ had taurodontism. ${ }^{2}$

True anodontia, or congenital absence of teeth, may be two types, total and partial. It is rare and associated commonly with ectodermal dysplasia. False anodontia results due to extraction of all teeth and also known as pseudo anodontia. True partial anodontia/oligodontia/hypodontia involves one or more teeth and third molars, maxillary lateral incisors and second premolars were commonly affected. In Down syndrome patients, Moraes et al. investigated the incidence of anodontia and it was found in 17 individuals with 44 involved teeth representing $39.2 \% .^{5}$

Hypodontia was more common in maxilla than mandible in Down syndrome children. The most common congenitally missing teeth in hypodontia were upper lateral incisors, upper second premolars, lower lateral incisors and lower second premolars and most prevalent in female population. ${ }^{1}$ Hypodontia is frequently associated with other anomalies including delayed tooth development, abnormal tooth morphology, such as microdontia, conical-crown shape, enamel hypoplasia, concomitant occurrence of supernumerary teeth. Cuoghi et al. observed 105 individuals with Down syndrome, out of them 17 individuals (16.19\%) had hypodontia. ${ }^{2}$ Andersson et al. conducted the study among 26 children with Down syndrome and hypodontia was found in 16 children (61.5\%). ${ }^{1}$ van Marrewijk et al. divided the group of 63 individuals with Down syndrome as a group with and without hypodontia. ${ }^{6}$ The group with hypodontia consisted of 38 subjects and $47.4 \%$ had missing teeth in both anterior and posterior parts of the jaws. ${ }^{6}$

van Marrewijk et al. investigated about the incidence of oligodontia among 63 individuals with Down syndrome and oligodontia was found in $23.8 \%$ of total study population. ${ }^{6}$

Singh et al. presented a case report which discussed about the coexistence of fusion and concrescence of primary in Down syndrome child and they observed the presence of fusion in relation to 61,62 and concrescence in relation to $81,82 .^{12}$

Moraes et al. investigated the incidence of conic teeth in 49 subjects with Down syndrome and it was found in 7 individuals (14.28\%) had conic teeth (Table 1). ${ }^{5}$

Tooth agenesis was present in $65 \%$ of individuals with Down syndrome ${ }^{8}$ and it was mostly bilateral commonly involving lateral incisors and second premolars in both maxilla and mandible. Tooth agenesis is a complex and multifactorial trait showing high prevalence in Down syndrome which include environmental and genetic factors and commonly affects maxillary lateral incisor, mandibular premolar and mandibular incisors. ${ }^{9}$ Gallo et al.

Table 1: Dental anomalies reported in literature

\begin{tabular}{|c|c|}
\hline Anomalies & References \\
\hline Anodontia & 2007, Moraes et al._-39.2\% \\
\hline \multirow[t]{2}{*}{ Taurodontism } & 2007, Moraes et al._-85.71\% \\
\hline & 2016, Cuoghi et al.- $9.52 \%$ \\
\hline \multirow[t]{2}{*}{ Microdontia } & 2007, Moraes et al.- $2.04 \%$ \\
\hline & 2016, Cuoghi et al.-16.19\% \\
\hline \multirow[t]{6}{*}{ Hypodontia } & 2013 , Andersson et al._-61.5\% \\
\hline & $\begin{array}{l}\text { 2015, van Marrewijk et al.- } \\
47.4 \%\end{array}$ \\
\hline & 2016, Cuoghi et al.-16.19\% \\
\hline & 2016, Palaska et al. \\
\hline & 2016, van der Lindern et al. \\
\hline & 2015, Reiche et al. \\
\hline Fissured lip and tongue & $\begin{array}{l}\text { 2015, Al-Maweri et al. }-64 \% \text { and } \\
78 \%\end{array}$ \\
\hline \multirow[t]{2}{*}{ Oligodontia } & $\begin{array}{l}\text { 2015, van Marrewijk et al.- } \\
23.8 \%\end{array}$ \\
\hline & 2015, Reiche et al. \\
\hline \multirow[t]{3}{*}{ Delayed eruption } & 2016, van de Linder et al. \\
\hline & 2016, Palaska et al. \\
\hline & 2017, Gallo et al. \\
\hline \multirow[t]{2}{*}{ Tooth agenesis } & 2017, Gallo et al._-65\% \\
\hline & 2016, Palaska et al. $-54.6-58.5 \%$ \\
\hline
\end{tabular}


conducted the study among 46 Down syndrome patients and tooth agenesis was present in $65 \%$ of Down syndrome patients. ${ }^{8}$ Palaska et al. observed $54.6-58.5 \%$ of Down syndrome individuals shows teeth agenesis, in which one quarter has one missing teeth, one third of them exhibit two missing teeth and remaining has three or more missing teeth. ${ }^{9}$

Abeleira et al. investigated about the dimension of tooth size among 40 individuals with Down syndrome using CBCT and it was concluded that the individuals with Down syndrome have smaller teeth than normal individuals. ${ }^{13}$

Tooth eruption may be delayed, may occur in an unusual order. The eruption movement results from a balance between tissue destruction and tissue formation. The delay in clinical emergence probably finds its origin in the gingival tissue or other factors such as the cellular processes at the apical and occlusal side of the erupting tooth. It is possible that a genetic disturbance in the RANKL/OPG system or the disturbance of RUNX2 is a cause for the impaired eruption. ${ }^{14}$ van der Linden et al. included 94 patients with Down syndrome in this study and the mean DMS (dental maturity score) for control children was 30.07 (SD 19.89) and for Down syndrome was 15.07 (SD 11.20). ${ }^{14}$

Microdontia is used to describe teeth which are smaller in size than normal teeth and is further classified into true generalized microdontia and relative generalized as called as "peg laterals" are also seen in Down syndrome patients. Cuoghi et al. evaluated 105 patients with Down syndrome and 17 of them (16.19\%) had microdontia. ${ }^{2}$ Moraes et al. investigated the incidence of anodontia and it was found only in 1 individual (2.04\%). ${ }^{5}$

Fissured tongue is a condition frequently seen in general population and is characterized by grooves that vary in depth noted along the dorsal and lateral aspect of tongue. It was commonly seen in Melkersson-Rosenthal syndrome and Down syndrome. Fissured tongue was more common in Down syndrome individuals compared to control subjects. ${ }^{3} \mathrm{Al}-$ Maweri et al. conducted a study among 50 individuals with Down syndrome and found that 39 of them (78\%) has fissured tongue and 32 individuals (64\%) has lip fissure. ${ }^{3}$ Open bite was one of the common finding in Down syndrome individuals which were due to macroglossia, where enlarged tongue interferes with the occlusion of the teeth.

\section{Conclusion}

The conclusion of this review is individuals with Down syndrome exhibit high prevalence of dental anomalies compared to normal individuals. The most prevalent dental anomalies among these individuals were taurodontism, anodontia, hypodontia, fissured tongue and teeth agenesis. These outcomes reinforce that dental care should be directed to prevent and/or control problems and to improve oral hygiene status of Down syndrome individuals.

\section{References}

1. Andersson EM, Axelsson S, Austeng ME, Øverland B, Valen IE, Jensen $\mathrm{TA}$, et al. Bilateral hypodontia is more common than unilateral hypodontia in children with Down syndrome: a prospective population-based study. Eur J Orthod 2013;36(4):414-418. DOI: 10.1093/ejo/cjt063.

2. Cuoghi OA, Topolski F, de Faria LP, Occhiena CM, Ferreira ND, Ferlin $\mathrm{CR}$, et al. Prevalence of dental anomalies in permanent dentition of brazilian individuals with Down syndrome. Open Dent J 2016;10: 469-473. DOI: 10.2174/1874210601610010469.

3. Al-Maweri SA, Tarakji B, Al-Sufyani GA, Al-Shamiri HM, Gazal G. Lip and oral lesions in children with Down syndrome. A controlled study. J Clin Exp Dent 2015;7(2):e284-e288. DOI: 10.4317/jced.52283.

4. Marques LS, Alcântara CE, Pereira LJ, Ramos-Jorge ML. Down syndrome: a risk factor for malocclusion severity? Braz Oral Res 2015;29:44. DOI: 10.1590/1807-3107BOR-2015.vol29.0044.

5. Moraes ME, Moraes LC, Dotto GN, Dotto PP, Santos LR. Dentalanomalies in patients with Down syndrome. Braz Dent J 2007;18(4):346-350. DOI: 10.1590/S0103-64402007000400014.

6. Van Marrewijk DJ, Van Stiphout MA, Reuland-Bosma W, Bronkhorst EM, Ongkosuwito EM. The relationship between craniofacial development and hypodontia in patients with Down syndrome. Eur J Orthod 2015;38(2):178-183. DOI: 10.1093/ejo/cjv054.

7. Phadke SR, Puri RD, Ranganath P. Prenatal screening for genetic disorders: suggested guidelines for the Indian scenario. Indian J Med Res 2017;146(6):689-699.

8. Gallo C, Pastore I, Beghetto M, Mucignat-Caretta C. Symmetry of dental agenesis in Down syndrome children. J Dent Sci 2019;14(1): 61-65. DOI: 10.1016/j.jds.2018.04.003.

9. Palaska PK, Antonarakis GS. Prevalence and patterns of permanent tooth agenesis in individuals with Down syndrome: a meta-analysis. Eur J Oral Sci 2016;124(4):317-328. DOI: 10.1111/eos.12282.

10. Reiche W, Párkányi L, Braunitzer G, Nagy K, Kárpáti K. Oligodontia in a teenager with Down syndrome-a case study. Klin Padiatr 2015;227(1):35-37. DOI: 10.1055/s-0034-1384525.

11. Durković J, Ubavić M, Durković M, Kis T. Prenatal screening markers for Down syndrome: sensitivity, specificity, positive and negative expected value method. J Med Biochem 2018;37(1):62-66.

12. Singh A, Bhatia HP, Sharma N. Coexistence of fusion and concrescence of primary teeth: in a child with Down syndrome. Spec Care Dent 2017;37(3):147-149. DOI: 10.1111/scd.12218.

13. Abeleira MT, Outumuro M, Ramos I, Limeres J, Diniz M, Diz P. Dimensions of central incisors, canines, and first molars in subjects with Down syndrome measured on cone-beam computed tomographs. Am J Orthod Dentofacial Orthop 2014;146(6):765-775. DOI: 10.1016/j.ajodo.2014.08.016.

14. Van der Linden MS, Vucic S, Van Marrewijk DJ, Ongkosuwito EM. Dental development in Down syndrome and healthy children: a comparative study using the Demirjian method. Orthod Craniofac Res 2017;20(2):65-70. DOI: 10.1111/ocr.12139. 